\title{
Phase II study of the paclitaxel, cisplatin, 5-fluorouracil and leucovorin (TPFL) regimen in the treatment of advanced or metastatic gastric cancer
}

\author{
JOO YOUNG JUNG ${ }^{1}$, JUNG HYE KWON ${ }^{1}$, JUNG HAN KIM ${ }^{1}$, HUN HO SONG ${ }^{1}$, INHO KIM ${ }^{2}$, KEUN SEOK LEE ${ }^{3}$, \\ HYO JUNG KIM ${ }^{1}$, DAE YOUNG ZANG ${ }^{1}$, JIN SEOK AHN ${ }^{4}$, JUNG-AE LEE ${ }^{5}$ and YOUNG-IEE PARK ${ }^{3}$ \\ ${ }^{1}$ Department of Internal Medicine, College of Medicine, Hallym University, Chuncheon; ${ }^{2}$ Department of Internal Medicine, \\ College of Medicine, Seoul National University, Seoul; ${ }^{3}$ National Cancer Center, Goyang; \\ ${ }^{4}$ Department of Internal Medicine, College of Medicine, Seongyunkwan University, Seoul; \\ ${ }^{5}$ Department of Internal Medicine, College of Medicine, Eulji University, Daejeon, Korea
}

Received August 20, 2008; Accepted November 18, 2008

DOI: $10.3892 /$ or_00000253

\begin{abstract}
Advanced or metastatic gastric cancer, which is one of the most common malignancies in Korea, is difficult to cure by surgery alone and generally requires combination chemotherapy. Paclitaxel is active against gastric cancer and when combined with 5-fluorouracil/leucovorin and/or cisplatin is effective in the treatment of gastric cancer. We attempted to determine the effect and safety with the combination of paclitaxel with split cisplatin and 5-fluorouracil/leucovorin in advanced or metastatic gastric cancer. Patients with histologically-proven locally advanced/metastatic or recurrent gastric cancer with an ECOG performance status 0-2 were enrolled. The patients received $135 \mathrm{mg} / \mathrm{m}^{2}$ of paclitaxel as a 3-h intravenous infusion on day 1 and 5fluorouracil $\left(1200 \mathrm{mg} / \mathrm{m}^{2}\right)$ plus leucovorin $\left(20 \mathrm{mg} / \mathrm{m}^{2}\right)$ as an intravenous infusion over $12 \mathrm{~h}$ plus cisplatin $\left(30 \mathrm{mg} / \mathrm{m}^{2}\right)$ by continuous intravenous infusion on days 1-3, every 21 days. Between September 2003 and April 2005, 30 patients (26 evaluable patients) with a median age of 57 years (range 34-74) were enrolled and underwent 111 completed treatment cycles (a median of 3 cycles per patient). Of the evaluable patients, 12 patients showed a partial response and 8 patients had stable disease. The overall response rate was $46.2 \%$. The median progression-free survival was 5.6 months $(95 \%$ CI. 3.76-7.4 months), and the median overall survival was 9.6 months (95\% CI. 6.67-12.47 months). The hematologic and non-hematologic toxicities were tolerable. The grade
\end{abstract}

Correspondence to: Dr Dae Young Zang, Department of Internal Medicine, Hallym University Sacred Heart Hospital, College of Medicine, Hallym University, 896, Pyeongchon-dong, Dongan-gu, Anyang, Gyeonggi-do 431-070, Korea

E-mail: fhdzang@hallym.or.kr

Key words: gastric cancer, paclitaxel, cisplatin, 5-fluorouracil, leucovorin
III and IV hematologic toxicities were anemia (6.8\%) and neutropenia $(2.6 \%)$. Febrile neutropenia was observed in 1 patients and 1 cycle. Other hematologic toxicities and grade III and IV non-hematologic toxicities, except nausea (66.7\%) and vomiting $(33.3 \%)$ were uncommon and not severe. TPFL combination chemotherapy is effective and tolerable with acceptable toxicities in patients with advanced/metastatic, recurrent gastric cancer.

\section{Introduction}

Gastric cancer is one of the leading causes of cancer death in Korea and Asian countries and is the most common malignant tumor in Korea (1). Surgery is the only effective treatment modality for localized resectable cases, but 65 to $75 \%$ of the patients with gastric cancer have advanced disease (1). The outcome of patients who present with metastatic or inoperable, locally advanced disease, as well as those with post-operative recurrence, is poor. The median survival time for such patients, if untreated, is $<5$ months (2). Chemotherapy has demonstrated a survival benefit and a positive impact on the quality of life for the patients with metastatic or unresectable gastric cancer according to many randomized studies (2). Thus, chemotherapy for advanced gastric cancer is now widely accepted. Although there is no generally accepted standard regimen, a number of chemotherapy regimens, such as 5-fluorouracil (5-FU), etoposide, and cisplatin, were developed in the past 3 decades and have shown an objective response rate of approximately 20-60\% (3-6). The combination chemotherapy regimens, usually based on 5-FU, have achieved good response rates, ranging from 20 to $40 \%(3,4)$. The activity and tolerability of the combination of 5-FU and cisplatin in patients with advanced gastric cancer have been confirmed in several studies $(5,6)$. Paclitaxel is an antimitotic agent with a unique cytotoxic mechanism affecting tubulin stabilization and polymerization, resulting in nonfunctional microtubules. This agent has been shown in vitro antitumor activity against various tumor cell lines, including gastric cancer (7). Ajani et al (8) and Cascinu et al (9) 
obtained $17-22 \%$ clinical response rates using paclitaxel as a single agent in patients with gastric cancer. Furthermore, paclitaxel-containing combinations, including 5-FU, cisplatin or etoposide yielded high response rates $(50 \%)$ with a median survival of 7-14 months (10-13).

$5-\mathrm{FU}$ acts synergistically with paclitaxel in a sequencedependent manner in which paclitaxel, followed by $5-\mathrm{FU}$, was highly synergistic, while pre-exposure to 5-FU resulted in antagonism against human gastric cancer cells (14). In addition, there was no overlapping toxicity profile between paclitaxel and infusional 5-FU. The combination of 5-FU/ leucovorin with paclitaxel have shown activity and tolerability against gastric cancer in some studies $(15,16)$.

Based on the activity of 5-FU and cisplatin in gastric cancer and on the meaningful results of the combination of 5-FU/leucovorin and paclitaxel, Honecker et al (10) conducted a clinical trial to determine the activity and toxicity of weekly paclitaxel and infusional 5-FU/leucovorin and cisplatin in gastric cancer, and demonstrated an overall response rate of $48 \%$ and good tolerablity. The hematologic toxicity was lower, however, peripheral neuropathy was higher than 3 weeks of paclitaxel.

Therefore, we conducted a phase II study to assess the efficacy and safety of the combination of paclitaxel, 5-FU/ leucovorin and cisplatin every 3 weeks in patients with gastric cancer to reduce peripheral neuropathy and lower hematologic toxicity with comparable response to weekly paclitaxel.

\section{Patients and methods}

Patients. Patients with histologically-proven advanced or metastatic gastric cancer were included in this study. Inclusion criteria were as follows: initially diagnosed disease, which was unresectable, locally-advanced or metastatic, or recurrent disease after curative resection; ECOG performance status $0-2$; presence of measurable disease; age between 18 and 75 years; life expectancy $>3$ months; no prior chemotherapy (except adjuvant chemotherapy without platinum agents or taxanes); adequate hematologic, renal and hepatic function, as defined by a granulocyte count $\geq 1.5 \times 10^{9} / 1$, thrombocytes $\geq 100 \times 10^{9} / 1$, serum creatinine $\leq 1.5 \mathrm{mg} / \mathrm{dl}$, bilirubin level $<2$-fold and liver enzymes $<3$-fold the upper normal limits; and written informed consent. All patients were required to be available for follow-up evaluation.

Patients were excluded from the study for the following reasons: active bleeding; a history of a secondary malignancy, except for non-melanomatous skin cancer or carcinoma in situ of the cervix; grade $\geq 2$ peripheral neuropathy according to the National Cancer Institute Common Toxicity Criteria (NCI-CTC); brain metastasis; concurrent insufficiently treated disease such as heart, renal or hepatic failure or uncontrolled infections; prior chemotherapy; presence of a concurrent psychiatric disorder; and pregnancy. Informed consent from all patients was obtained before they entered the study.

Treatment plans. On day $1,135 \mathrm{mg} / \mathrm{m}^{2}$ of paclitaxel was intravenously administered in $250 \mathrm{ml}$ of normal saline as a 3-h infusion. Within $2 \mathrm{~h}$ after completion of paclitaxel, $20 \mathrm{mg} / \mathrm{m}^{2}$ of leucovorin was administered as an intravenous bolus injection, which was followed by both $1200 \mathrm{mg} / \mathrm{m}^{2}$ of 5-FU as an intravenous infusion over $12 \mathrm{~h}$ and $30 \mathrm{mg} / \mathrm{m}^{2}$ of cisplatin as a continuous intravenous infusion via a separate line. Leucovorin, 5-FU and cisplatin was given also on day 2 and 3 . The treatment was repeated every 21 days. To avoid hypersensitivity reactions involving paclitaxel, we gave dexamethasone $(20 \mathrm{mg}$ at $1 \mathrm{~h}$ ), ranitidine $(300 \mathrm{mg}$ at $30 \mathrm{~min})$ and diphenylhydramine $(50 \mathrm{mg}$ at $30 \mathrm{~min}$ ) before paclitaxel. All patients also received adequate antiemetic premedication prior to chemotherapy. Granulocyte colonystimulating factor (G-CSF) was not prophylactically administered. Dose escalation after reduction was not allowed. Chemotherapy was given until the occurrence of disease progression, unacceptable toxicity or treatment withdrawal. The treatment continued for a maximum of 6 cycles.

Response and toxicity evaluation. Prior to therapy, baseline evaluation of each patient which included a clinical history and physical examination, a complete blood count and serum chemistries (including liver and kidney function tests), and an electrocardiogram were obtained. A radiologic evaluation was completed within 4 weeks prior to the treatment. During treatment, physical examination, performance status, a complete blood count and serum chemistries were recorded prior to each subsequent cycle. Radiologic studies, including computed tomography (CT), of the measurable lesion(s) were repeated every two cycles.

Treatment response was evaluated every 2 cycles according to the guideline of the Response Evaluation Criteria in Solid Tumors (RECIST) committee. Patients were considered as assessable for response if they showed evidence of early disease progression clinically or radiologically within 2 cycles, or if they had received a minimum of 2 cycles of treatment with at least one tumor measurement. A measurable lesion was defined as $10 \mathrm{~mm}$ in the longest dimension, assessed by a spiral CT scan. If a patient was documented as having a complete (CR) or a partial response (PR), the response was confirmed at least 4 weeks after the first evident response.

Progression-free survival (PFS) was defined as the time elapsed from the start of treatment until disease progression or death of any cause, and overall survival (OS) was defined from the start of treatment to death.

All patients were evaluated for toxicity from the time of the first cycle. Toxicity was evaluated as a grade according to the NCI-CTC (version 2.0).

Study endpoints/statistical consideration. The primary endpoint of this study was overall response rate (ORR; CR + PR), and the secondary endpoints were PFS, OS, disease control rate (DCR) and safety. Survival was assessed on an intention to treat basis. Time-dependent variables were estimated using the Kaplan-Meier method.

\section{Results}

Patient characteristics. Between September 2003 and September 2005, 30 patients were enrolled in this study. All patients were assessable for survival parameters and toxicity and 26 of the patients were evaluable for tumor response. Four patients were excluded from analysis because they 
Table I. Patient characteristics.

\begin{tabular}{|c|c|}
\hline & No. of patients $(\%)$ \\
\hline Total enrolled patients & 30 \\
\hline Evaluable patients & 26 \\
\hline Age, years (Median range) & $57(34-74)$ \\
\hline \multicolumn{2}{|l|}{ Gender } \\
\hline Male & $24(80)$ \\
\hline Female & $6(20)$ \\
\hline \multicolumn{2}{|l|}{$\mathrm{ECO}$} \\
\hline 0 & $6(20)$ \\
\hline 1 & $17(56.7)$ \\
\hline 2 & $7(23.3)$ \\
\hline \multicolumn{2}{|l|}{ Histology (adenocarcinoma) } \\
\hline Well differentiated & $2(6.7)$ \\
\hline Moderately differentiated & $5(16.7)$ \\
\hline Poorly differentiated & $7(23.3)$ \\
\hline Unspecified & $16(53.3)$ \\
\hline \multicolumn{2}{|l|}{ Previous gastrectomy } \\
\hline Yes & $13(43.3)$ \\
\hline No & $7(23.3)$ \\
\hline \multicolumn{2}{|l|}{ Disease site } \\
\hline Peritoneum & $6(20)$ \\
\hline Pleura & $1(3.3)$ \\
\hline Liver & $12(40)$ \\
\hline Lung & $1(3.3)$ \\
\hline Lymph node & $11(36.7)$ \\
\hline Ovary & $1(3.3)$ \\
\hline Total & $26(86.7)$ \\
\hline \multicolumn{2}{|l|}{ Chemotherapy status } \\
\hline Chemonaive & 20 \\
\hline Adjuvant chemotherapy & 10 \\
\hline Oral doxifluridine & 2 \\
\hline 5-FU + mitomycin $\mathrm{C}$ & 8 \\
\hline
\end{tabular}

did not complete the minimum 2 cycles and they withdrew their consent after the first cycle without any definite evidence of disease progression or adverse events. The characteristics of the patients are given in Table I. The median age of the patients was 57 years (range, 34-74 years). The study group included 24 males and 6 females, and the majority of patients $(57.3 \%)$ had a performance status of 1, according to the ECOG scale. Most of the patients [26 (86.7\%)] had metastatic disease, and the liver [12 of 30 patients (40\%)] and lymph nodes [11 of 30 patients $(36.7 \%)$ ] were the most common metastatic sites. All patients had measurable tumor lesions. Thirteen patients $(43.3 \%)$ had undergone gastrectomy prior to chemotherapy.

Efficacy. Twenty-six patients were evaluable for tumor responses. There were no CRs and 12 patients achieved a $\mathrm{PR}$, giving an ORR of $46.2 \%$ [95\% confidence interval
Table II. Response (n=26) and survival $(n=30)$.

\begin{tabular}{lc}
\hline & No. of patients (\%) \\
\hline Response & \\
CR & 0 \\
PR & $12(46.2)$ \\
SD & $8(30.8)$ \\
PD & $6(23.1)$ \\
Survival & \\
Median OS & \\
Median PFS & 5.6 months (95\% CI 6.67-12.47) \\
\hline
\end{tabular}

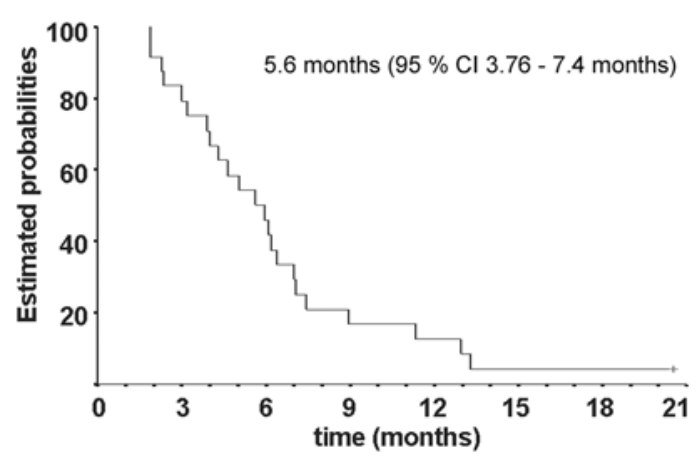

Figure 1. Progression-free survival $(\mathrm{n}=26)$.

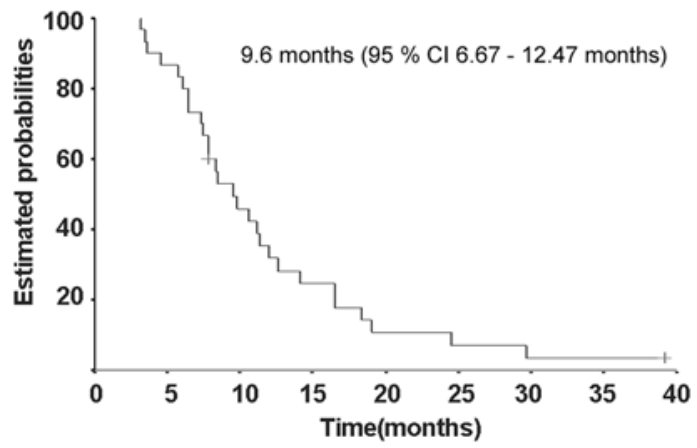

Figure 2. Overall survival $(\mathrm{n}=30)$.

(CI), 30.7-61.7)]. Stable disease was obtained in 8 patients $(30.8 \%)$ and progressive disease was observed in 6 patients (23.1\%). Therefore, the overall disease control rate (DCR) was $77.0 \%$ (95\% CI 67.5-86.5; Table II).

All patients were included in the survival analysis on an intent to treat basis. The median follow-up period was 9.0 months (range, 2.5-39.2 months). At the time of analysis, the median PFS was 5.6 months (95\% CI 3.76-7.40 months) and the OS was 9.6 months (95\% CI 6.67-12.47 months). The Kaplan-Meier estimated PFS and OS curve are shown in Figs. 1 and 2, respectively. 
Table III. Severity and incidence of toxicity.

Grade 1 Grade 2 Grade 3 Grade 4

\begin{tabular}{lcccc}
\hline $\begin{array}{l}\text { Hematologic } \\
\text { toxicity }\end{array}$ & \multicolumn{5}{c}{ No. of cycles $(\mathrm{n}=117)(\%)$} \\
Anemia & $19(16.2)$ & $34(29.0)$ & $8(6.8)$ & - \\
Neutropenia & $16(13.7)$ & $10(8.5)$ & $3(2.6)$ & - \\
Thrombocytopenia & $9(7.7)$ & - & - & -
\end{tabular}

$\begin{array}{lllcl}\begin{array}{l}\text { Non-hematologic } \\ \text { toxicity } \\ \text { Nausea }\end{array} & 9(30.0) & 11(36.7) & - & - \\ \text { Vomiting } & 4(13.3) & 6(20.0) & - & - \\ \text { Diarrhea } & 3(10.0) & 3(10.0) & 1(3.3) & - \\ \text { Stomatitis } & 5(16.7) & 1(3.3) & - & - \\ \text { Neuropathy } & 2(6.7) & 4(13.3) & - & - \\ \text { Alopecia } & 3(10.0) & 7(23.3) & 2(6.6) & - \\ \text { Renal } & 3(10.0) & 2(6.6) & - & - \\ \text { Hypertrans- } & 1(3.3) & - & - & - \\ \text { aminasemia } & & & & - \\ \end{array}$

Toxicity. Overall, 117 cycles were administered to the 26 patients (median, 4 cycles per patient; range, 1-6 cycles). The median dose intensity of 5-FU, paclitaxel, and cisplatin was $3600 \mathrm{mg} / \mathrm{m}^{2} /$ cycle, $135 \mathrm{mg} / \mathrm{m}^{2} /$ cycle, and $90 \mathrm{mg} / \mathrm{m}^{2}$ / cycle, respectively and an average dose intensity of this regimen was 0.98 (range, 0.83-1.0).

The occurrence and the incidence of toxicities are shown in Table III. There were no cases of grade 4 toxicity. The most common toxicities were gastrointestinal. The most common non-hematologic toxicity was nausea, and grade 1 and 2 nausea, vomiting, diarrhea, and stomatitis were reported in $66.7,33.3,20$, and $20 \%$, respectively. Grade 3 non-hematolgic toxicities were uncommon and included diarrhea $(3.3 \%)$ and alopecia $(6.6 \%)$. The most common grade 3 hematologic toxicity was anemia, which occurred 8 times $(6.8 \%)$, and grade 3 neutropenia, which occured 3 times $(2.6 \%)$. Febrile neutropenia was observed in one patient in one cycle and recovered without complications. There were no treatment-related deaths in this trial.

\section{Discussion}

The results of this phase II study indicate that the TPFL regimen is effective and safe for the palliative treatment of advanced, metastatic or recurrent gastric cancer, and has excellent tolerability.

In advanced or metastatic gastric cancer, systemic chemotherapy has been attempted for a palliative treatment, leading to improvement of tumor responses, quality of life, and survival compared to best supportive case (6). In phase II trials, response rates of 30 to $70 \%$ have been reported for major protocols such as 5-FU and cisplatin (FP) (6), 5-FU, leucovorin, and cisplatin (FLP) (17), 5-FU, doxorubicin and mitomycin C (FAM) $(3,6), 5-\mathrm{FU}$, doxorubicin, and methotrexate (FAMTX) $(3,18)$, etoposide, doxorubicin, and cisplatin (EAP) (19), epirubicin, cisplatin and 5-FU continuous infusion (ECF) (20), and oxaliplatin-based regimens (21). However, the results of the subsequent phase III trials have often failed to confirm the relatively high response rates of earlier reports $(6,18,22)$. Furthermore, the survival benefit was limited, with substantial treatment-related toxicities in most trials. Therefore, the standard chemotherapy has not yet been established. In Europe, anthracycline-containing regimens, such as ECF $(18,22)$, have been used as a reference, while 5-FU plus cisplatin or 5-FU plus cisplatin or 5-FU alone are preferred in Asia and the USA (6).

Recently, several new agents have been included in third-generation regimens in gastric cancer including a topoisomerase I inhibitor (CPT-11) or taxanes (docetaxel and paclitaxel). Among these new agents, docetaxel was the first agent which was tried in advanced gastric cancer, showing an improved efficacy in phase II trials (23-25). The combination of docetaxel, cisplatin and 5-FU (DCF) showed better efficacy than FP in terms of tumor response and survival in the V325 trial (24). However, severe hematologic toxicities were observed in $>80 \%$ of patients with DCF in V325. These findings are consistent with other trials involving docetaxel and $71 \%$ of grade $3-4$ neutropenias including $12 \%$ of febrile neutropenias, was reported, even with the prophylactic administration of GCSF in other phase II trial (25). Thus, the docetaxel combination shows improved efficacy, but also substantial hematologic toxicity.

Paclitaxel, another taxane, has a wide range of antitumor activities and a synergistic effect with other various chemotherapeutic agents (cisplatin and 5-FU) in combination therapy in gastric cancer. In combination with 5-FU and/or platinum compounds, response rates ranging from 32 to $65 \%$ were reported in many trials conducted in different settings $(10,11,26-30)$; paclitaxel was delivered weekly or every 3 weeks. In other studies, cisplatin or carboplatin in combination with paclitaxel and 5-FU (intravenous or oral fluoroyrimidine) were administered; the subjects were chemonaive patients and/or previously treated patients. However, relatively high incidences $(14-45 \%)$ of grade 3 or 4 neutropenias were significant adverse effects in these studies $(11,15,26,28,29)$.

Continuous infusion of 5-FU combined with leucovorin has been an acceptable regimen in gastrointestinal malignancies, because this combination regimen is less toxic and relatively more effective. It was evaluated mainly as a salvage therapy for patients with gastric cancer, even those in poor general condition, such as acute disseminated intravascular coagulation $(31,32)$. In this study, we chose paclitaxel as a mainstay agent and combined 5-FU/leucovorin and continuous cisplatin. The nationale behind this strategy was: i) the synergistic effect of the combination; ii) no overlapping toxicity; iii) recent results of 5-FU, cisplatin and paclitaxel combination (11); and iv) less myelotoxicity of paclitaxel than docetaxel $(30,33)$.

The ORR of $46.2 \%$ (95\% CI, 30.7-61.7\%) and the median OS of 9.6 months (95\% CI, 6.67-12.47 months) in this study were within the ranges [30-70\% (5.5-11 months)] obtained by previously reported major protocols such as FP (6), FLP 
(17), FAM $(3,6)$, FAMTX $(3,18)$, ECF $(20)$, and oxaliplatinbased (21).

There was no additional benefit in increasing paclitaxel dose over $175 \mathrm{mg} / \mathrm{m}^{2}$ with respect to tumor response and survival in a phase III trial (34). Moreover, paclitaxel binding site plasma steady-state concentrations achieved by dose $\geq 135 \mathrm{mg} / \mathrm{m}^{2}$ (24-h infusion), and cytotoxicity was observed to plateau in vitro on increasing the paclitaxel concentration (35). On the other hand, the hematologic toxicity, especially neutropenia, was aggravated with increasing dose of paclitaxel. In order to increase the dose intensity, yet at the same time to reduce hematologic toxicity, Honecker et al used paclitaxel at a weekly dose of $80 \mathrm{mg} / \mathrm{m}^{2}$, instead of a dose of $175 \mathrm{mg} / \mathrm{m}^{2}$, every 3 weeks (10). In this study, we chose the dose of a paclitaxel dose of $135 \mathrm{mg} / \mathrm{m}^{2}$ every 3 weeks to reduce hematologic toxicity with comparable efficacy. As a result, the ORR and median OS were comparable to the results of the previous studies $(10,15,27,33)$ and grade 3 neutropenia was observed in only $2.6 \%$ with no treatment-related deaths.

Peripheral neuropathy is the most troublesome toxicity for patients receiving paclitaxel-containing regimens, and paclitaxel dose intensity is related to the occurrence of severe neurologic toxicities, especially when combined with cisplatin, therefore cisplatin is commonly replaced with carboplatin. However, the paclitaxel/carboplatin regimen causes more myelosuppression. In this study, peripheral neuropathy developed in $20 \%$ of patients receiving low-dose paclitaxel and split-dose cisplatin without grade 3-4 neuropathies. This frequency of peripheral neuropathy for low-dose paclitaxel/ cisplatin regimen is lower than paclitaxel and carboplatin regimens, which showed 50-85\% peripheral neuropathies of all grades and $0-8 \%$ grade 3 or 4 peripheral neuropathies, respectively (29). No patients discontinued protocol treatment due to paclitaxel-related neuropathy.

Cisplatin is an active drug and used for the treatment of gastric cancer, especially as a component of combination treatment. However, the use of cisplatin has been limited because of its toxicities, such as renal toxicity, neurotoxicity and high emetogenic potential. Belliveau et al (36) have demonstrated that the area under the curve of the concentration of non-protein-bound cisplatin, which is related to the antitumor activity of cisplatin, was substantially higher in patients given a continuous infusion of cisplatin than after similar doses given as a bolus. Moreover, Drewinko et al (37) have suggested that a protracted infusion of low dose cisplatin provides better antitumor effects. With respect to toxicities, the acute nausea and vomiting are dramatically reduced by daily continuous infusion of cisplatin without reduction of antitumor activity (38). There have been many attempts to reduce toxicities and increase the dose intensity of cisplatin (39-41). We used a continuous infusion of cisplatin for 3 days at a dose of $30 \mathrm{mg} / \mathrm{m}^{2}$ every 3 weeks. Nausea is the most common non-hematologic complication (66.7\%) and vomiting was observed in $33.3 \%$ of the patients, which was not uncommon but manageable. However, grade 3 or 4 nausea and vomiting was not observed. Renal toxicity associated with cisplatin, was uncommon $(16.6 \%)$ in this study.

Advanced gastric cancer includes considerable proportion of non-measurable diseases such as peritoneal seeding or gastric mass. In recent reports, the most frequent site of recurrence in gastric cancer was demonstrated as peritoneal seeding, especially in an Asian population (42). In addition, the prognosis was different between measurable and nonmeasurable disease (43). It is well-known that, in the longterm, those who usually respond to the treatment will do better than the others, while the clinical response is not a valid surrogate of survival (44). On the other hand, the PFS can reflect tumor growth and be assessed before the determination of a survival benefit without confounding by subsequent therapy (45). Therefore, we also agree with the theory that PFS can reflect the chemotherapy benefit more precisely. Recently, the number of studies in which PFS was used as the primary endpoint has been increasing, which needs more trials to evaluate the adequacy of PFS as the primary goal in phase II trial. The median PFS interval of 5.6 months in this study was comparable with those observed in other studies $(10-12,15)$.

The benefit-to-risk ratio might be very important issue in the treatment of advanced gastric cancer. Although several chemotherapy regimens have been introduced, the benefit in survival or response appears to be marginal but the treatment-related toxicities are usually inevitable $(46,47)$. In addition, there is no consensus on a strategy for the sequential use of chemotherapy for advanced/metastatic or recurrent gastric cancer, such as the recommendation of aggressive chemotherapy for the first-line setting or the reservation of strong chemotherapy for salvage chemotherapy. Although there is no rule, we treat patients with gastric cancer using an individualized approach. It seems to be reasonable to use more aggressive regimens to gain longer survival for fit patients. On the other hand, it might be better to use drugs without severe toxic effects to achieve palliative goal for unfit patients who are elderly or have poor performance status. Therefore, the combination of paclitaxel, infusional 5FU/leucovorin and continous cisplatin, which was used in this study, is a meaningful therapeutic option, especially in elderly patients or those in poor condition.

In conclusion, the combination of paclitaxel and 5-FU/ leucovorin and continuous split-dose cisplatin (TPFL) appears to be an active chemotherapy regimen with high tolerability, suggesting the use of TPFL regimen as an alternative modality of treatment for patients with advanced/ metastatic or recurrent gastric cancer, especially in patients with relatively poor performance status. These results need to confirm the efficacy of this regimen and to determine the optimal paclitaxel dose and combination schedule to improve treatment outcome in further trials.

\section{References}

1. Lee HJ, Yang HK and Ahn YO: Gastric cancer in Korea. Gastric Cancer 5: 177-182, 2002.

2. Glimelius B, Ekstrom K, Hoffman K, et al: Randomized comparison between chemotherapy plus best supportive care with best supportive care in advanced gastric cancer. Ann Oncol 8: 163-168, 1997.

3. Wils JA, Klein HO, Wagener DJ, et al: Sequential high-dose methotrexate and fluorouracil combined with doxorubicin - a step ahead in the treatment of advanced gastric cancer: a trial of the European Organization for Research and Treatment of Cancer Gastrointestinal Tract Cooperative Group. J Clin Oncol 9: $827-831,1991$. 
4. Schipper DL and Wagener DJ: Chemotherapy of gastric cancer. Anticancer Drugs 7: 137-149, 1996.

5. Okada Y, Anai H, Hattori T, Maehara Y, Nishimura J, Sugimachi $\mathrm{K}$ and Nawata H: Cisplatin plus continuous infusion of 5-fluorouracil for 5 days effective for patients with advanced gastric cancer. Anticancer Drugs 2: 453-456, 1991.

6. Kim NK, Park YS, Heo DS, et al: A phase III randomized study of 5-fluorouracil and cisplatin versus 5-fluorouracil, doxorubicin, and mitomycin $\mathrm{C}$ versus 5-fluorouracil alone in the treatment of advanced gastric cancer. Cancer 71: 3813-3818, 1993.

7. Chang YF, Li LL, Wu CW, Liu TY, Lui WY, P'eng FK and Chi CW: Paclitaxel-induced apoptosis in human gastric carcinoma cell lines. Cancer 77: 14-18, 1996.

8. Ajani JA, Fairweather J, Dumas P, Patt YZ, Pazdur R and Mansfield PF: Phase II study of taxol in patients with advanced gastric carcinoma. Cancer J Sci Am 4: 269-274, 1998.

9. Cascinu S, Graziano F, Cardarelli N, Marcellini M, Giordani P, Menichetti ET and Catalano G: Phase II study of paclitaxel in pretreated advanced gastric cancer. Anticancer Drugs 9: 307-310, 1998.

10. Honecker F, Kollmannsberger C, Quietzsch D, et al: Phase II study of weekly paclitaxel plus 24-h continuous infusion 5fluorouracil, folinic acid and 3-weekly cisplatin for the treatment of patients with advanced gastric cancer. Anticancer Drugs 13: 497-503, 2002.

11. Kim YH, Shin SW, Kim BS, et al: Paclitaxel, 5-fluorouracil, and cisplatin combination chemotherapy for the treatment of advanced gastric carcinoma. Cancer 85: 295-301, 1999.

12. Kollmannsberger C, Quietzsch D, Haag C, et al: A phase II study of paclitaxel, weekly, 24-h continous infusion 5fluorouracil, folinic acid and cisplatin in patients with advanced gastric cancer. Br J Cancer 83: 458-462, 2000.

13. Lokich JJ, Sonneborn H, Anderson NR, Bern MM, Coco FV, Dow E and Oliynyk P: Combined paclitaxel, cisplatin, and etoposide for patients with previously untreated esophageal and gastroesophageal carcinomas. Cancer 85: 2347-2351, 1999.

14. Grem JL, Nguyen D, Monahan BP, Kao V and Geoffroy FJ: Sequence-dependent antagonism between fluorouracil and paclitaxel in human breast cancer cells. Biochem Pharmacol 58: 477-486, 1999.

15. Bokemeyer C, Hartmann JT, Lampe CS, Clemens MR, Quietzsch D, Forkmann L and Kanz L: Paclitaxel and weekly 24-h infusion of 5-fluorouracil/folinic acid in advanced gastric cancer. Semin Oncol 24: S19-96-S19-100, 1997.

16. Cho BC, Kim JH, Kim CB, Sohn JH, Choi HJ, Lee YC and Ahn JB: Paclitaxel and leucovorin-modulated infusional 5fluorouracil combination chemotherapy for metastatic gastric cancer. Oncol Rep 15: 621-627, 2006.

17. Ychou M, Astre C, Rouanet P, et al: A phase II study of 5fluorouracil, leucovorin and cisplatin (FLP) for metastatic gastric cancer. Eur J Cancer 32A: 1933-1937, 1996.

18. Vanhoefer U, Rougier P, Wilke H, et al: Final results of a randomized phase III trial of sequential high-dose methotrexate, fluorouracil, and doxorubicin versus etoposide, leucovorin, and fluorouracil versus infusional fluorouracil and cisplatin in advanced gastric cancer: a trial of the european organization for research and treatment of cancer gastrointestinal tract cancer cooperative group. J Clin Oncol 18: 2648-2657, 2000.

19. Preusser P, Wilke H, Achterrath W, et al: Phase II study with the combination etoposide, doxorubicin, and cisplatin in advanced measurable gastric cancer. J Clin Oncol 7: 1310-1317, 1989.

20. Zaniboni A, Barni S, Labianca R, et al: Epirubicin, cisplatin, and continuous infusion 5-fluorouracil is an active and safe regimen for patients with advanced gastric cancer: an italian group for the study of digestive tract cancer (GISCAD) report. Cancer 76: 1694-1699, 1995.

21. Louvet C, Andre T, Tigaud JM, et al: Phase II study of oxaliplatin, fluorouracil, and folinic acid in locally advanced or metastatic gastric cancer patients. J Clin Oncol 20: 4543-4548, 2002.

22. Webb A, Cunningham D, Scarffe JH, et al: Randomized trial comparing epirubicin, cisplatin, and fluorouracil versus fluorouracil, doxorubicin, and methotrexate in advanced esophagogastric cancer. J Clin Oncol 15: 261-267, 1997.
23. Constenla M, Garcia-Arroyo R, Lorenzo I, Carrete N, Campos B and Palacios P: Docetaxel, 5-fluorouracil, and leucovorin as treatment for advanced gastric cancer: results of a phase II study. Gastric Cancer 5: 142-147, 2002.

24. Van Cutsem E, Moiseyenko VM, Tjulandin S, et al: Phase III study of docetaxel and cisplatin plus fluorouracil compared with cisplatin and fluorouracil as first-line therapy for advanced gastric cancer: a report of the V325 study group. J Clin Oncol 24: 4991-4997, 2006.

25. Jeung HC, Rha SY, Kim YT, Noh SH, Roh JK and Chung HC: A phase II study of infusional 5-fluorouracil and low-dose leucovorin with docetaxel for advanced gastric cancer. Oncology 70: 63-70, 2006

26. Kim JG, Sohn SK, Song HS, et al: Multicenter phase II study of weekly paclitaxel plus cisplatin combination chemotherapy in patients with advanced gastric cancer. Cancer Chemother Pharmacol 60: 863-869, 2007.

27. Bokemeyer C, Lampe CS, Clemens MR, et al: A phase II trial of paclitaxel and weekly 24-h infusion of 5-fluorouracil/folinic acid in patients with advanced gastric cancer. Anticancer Drugs 8: 396-399, 1997

28. Chao Y, Li CP, Chao TY, et al: An open, multi-centre, phase II clinical trial to evaluate the efficacy and safety of paclitaxel, UFT, and leucovorin in patients with advanced gastric cancer. Br J Cancer 95: 159-163, 2006.

29. Gadgeel SM, Shields AF, Heilbrun LK, Labadidi S, Zalupski M, Chaplen R and Philip PA: Phase II study of paclitaxel and carboplatin in patients with advanced gastric cancer. Am J Clin Oncol 26: 37-41, 2003.

30. Murad AM, Petroianu A, Guimaraes RC, Aragao BC, Cabral LO and Scalabrini-Neto AO: Phase II trial of the combination of paclitaxel and 5-fluorouracil in the treatment of advanced gastric cancer: a novel, safe and effective regimen. Am J Clin Oncol 22: 580-586, 1999.

31. Vanhoefer U, Wilke H, Weh HJ, et al: Weekly high-dose 5fluorouracil and folinic acid as salvage treatment in advanced gastric cancer. Ann Oncol 5: 850-851, 1994.

32. Johnson PW, Thompson PI, Seymour MT, Deasy NP, Thuraisingham RC, Slevin ML and Wrigley PF: A less toxic regimen of 5-fluorouracil and high-dose folinic acid for advanced gastrointestinal adenocarcinomas. Br J Cancer 64: 603-605, 1991

33. Yeh KH, Lu YS, Hsu CH, et al: Phase II study of weekly paclitaxel and 24-h infusion of high-dose 5-fluorouracil and leucovorin in the treatment of recurrent or metastatic gastric cancer. Oncology 69: 88-95, 2005.

34. Omura GA, Brady MF, Look KY, et al: Phase III trial of paclitaxel at two dose levels, the higher dose accompanied by filgrastim at two dose levels in platinum-pretreated epithelial ovarian cancer: an intergroup study. J Clin Oncol 21: 2843-2848, 2003.

35. Rowinsky EK, Jiroutek M, Bonomi P, Johnson D and Baker SD: Paclitaxel steady-state plasma concentration as a determinant of disease outcome and toxicity in lung cancer patients treated with paclitaxel and cisplatin. Clin Cancer Res 5: 767-774, 1999.

36. Belliveau JF, Posner MR, Ferrari L, et al: Cisplatin administered as a continuous 5-day infusion: plasma platinum levels and urine platinum excretion. Cancer Treat Rep 70: 1215-1217, 1986.

37. Drewinko B, Brown BW and Gottlieb JA: The effect of cisdiamminedichloroplatinum (II) on cultured human lymphoma cells and its therapeutic implications. Cancer Res 33: 3091-3095, 1973.

38. Salem P, Hall SW, Benjamin RS, Murphy WK, Wharton JT and Bodey GP: Clinical phase I-II study of cis-dichlorodiammineplatinum(II) given by continuous lv infusion. Cancer Treat Rep 62: 1553-1555, 1978 .

39. Salem P, Khalyl M, Jabboury K and Hashimi L: Cis-diamminedichloroplatinum (II) by 5-day continuous infusion: a new dose schedule with minimal toxicity. Cancer 53: 837-840, 1984.

40. Posner MR, Ferrari L, Belliveau JF, et al: A phase I trial of continuous infusion cisplatin. Cancer 59: 15-18, 1987

41. Chi KH, Chan WK, Shu CH, Law CK, Chen SY, Yen SH and Chen KY: Elimination of dose limiting toxicities of cisplatin, 5-fluorouracil, and leucovorin using a weekly 24-h infusion schedule for the treatment of patients with nasopharyngeal carcinoma. Cancer 76: 2186-2192, 1995.

42. Yoo CH, Noh SH, Shin DW, Choi SH and Min JS: Recurrence following curative resection for gastric carcinoma. Br J Surg 87: 236-242, 2000. 
43. Shah MA, Ramanathan RK, Ilson DH, et al: Multicenter phase II study of irinotecan, cisplatin, and bevacizumab in patients with metastatic gastric or gastroesophageal junction adenocarcinoma. J Clin Oncol 24: 5201-5206, 2006.

44. Weiss GB, Bunce H III and Hokanson JA: Comparing survival of responders and non-responders after treatment: a potential source of confusion in interpreting cancer clinical trials. Control Clin Trials 4: 43-52, 1983.

45. Rothenberg ML, Carbone DP and Johnson DH: Improving the evaluation of new cancer treatments: challenges and opportunities. Nat Rev Cancer 3: 303-309, 2003.
46. Wagner AD, Grothe W, Haerting J, Kleber G, Grothey A and Fleig WE: Chemotherapy in advanced gastric cancer: a systematic review and meta-analysis based on aggregate data. J Clin Oncol 24: 2903-2909, 2006.

47. Pinto C, Di Fabio F, Siena S, et al: Phase II study of cetuximab in combination with FOLFIRI in patients with untreated advanced gastric or gastroesophageal junction adenocarcinoma (FOLCETUX study). Ann Oncol 18: 510-517, 2007. 